\title{
Decentralizing Attribute-Based Encryption
}

\author{
Allison Lewko ${ }^{\star}$ and Brent Waters ${ }^{\star \star}$ \\ University of Texas Austin \\ \{alewko, bwaters\}@cs.utexas.edu
}

\begin{abstract}
We propose a Multi-Authority Attribute-Based Encryption (ABE) system. In our system, any party can become an authority and there is no requirement for any global coordination other than the creation of an initial set of common reference parameters. A party can simply act as an ABE authority by creating a public key and issuing private keys to different users that reflect their attributes. A user can encrypt data in terms of any boolean formula over attributes issued from any chosen set of authorities. Finally, our system does not require any central authority.

In constructing our system, our largest technical hurdle is to make it collusion resistant. Prior Attribute-Based Encryption systems achieved collusion resistance when the ABE system authority "tied" together different components (representing different attributes) of a user's private key by randomizing the key. However, in our system each component will come from a potentially different authority, where we assume no coordination between such authorities. We create new techniques to tie key components together and prevent collusion attacks between users with different global identifiers.

We prove our system secure using the recent dual system encryption methodology where the security proof works by first converting the challenge ciphertext and private keys to a semi-functional form and then arguing security. We follow a recent variant of the dual system proof technique due to Lewko and Waters and build our system using bilinear groups of composite order. We prove security under similar static assumptions to the LW paper in the random oracle model.
\end{abstract}

\section{Introduction}

Traditionally, we view encryption as a mechanism for a user, Alice, to confidentially encode data to a target recipient, Bob. Alice encrypts the data under the recipient's public key such that only Bob, with knowledge of his private key, can decrypt it.

\footnotetext{
* Supported by National Defense Science and Engineering Graduate Fellowship.

** Supported by NSF CNS-0915361, and CNS-0952692, the MURI program under AFOSR Grant No: FA9550-08-1-0352. Department of Homeland Security Grant 2006-CS-001-000001-02 (subaward 641), a Google Faculty Research award, and the Alfred P. Sloan Foundation.
} 
However, in many applications, we find we need to share data according to an encryption policy without prior knowledge of who will be receiving the data. Suppose an administrator needs to encrypt a junior faculty member's performance review for all senior members of the computer science department or anyone in the dean's office. The administrator will want to encrypt the review with the access policy ("Computer Science" AND "Tenured") OR "Dean's OfFICE". In this system, only users with attributes (credentials) that match this policy should be able to decrypt the document. The key challenge in building such systems is to realize security against colluding users. For instance, the encrypted records should not be accessible to a pair of unauthorized users, where one has the two credentials of "TEnURED" and "ChEMISTRY" and the other one has the credential of "COMPUTER SCIENCE". Neither user is actually a tenured faculty member of the Computer Science Department.

Sahai and Waters [45] proposed a solution to the above problem that they called Attribute-Based Encryption (ABE). In an ABE system, a party encrypting data can specify access to the data as a boolean formula over a set of attributes. Each user in the system will be issued a private key from an authority that reflects their attributes (or credentials). A user will be able to decrypt a ciphertext if the attributes associated with their private key satisfy the boolean formula ascribed to the ciphertext. A crucial property of ABE systems is that they resist collusion attacks as described above.

Since the introduction of Attribute-Based Encryption, several works 83044429 23154/2122137] have proposed different ABE systems and applications. In almost all $\mathrm{ABE}$ proposals, private keys were issued by one central authority that would need to be in a position to verify all the attributes or credentials it issued for each user in the system. These systems can be utilized to share information according a policy over attributes issued within a domain or organization, however, in many applications a party will want to share data according to a policy written over attributes or credentials issued across different trust domains and organizations. For instance, a party might want to share medical data only with a user who has the attribute of "Doctor" issued by a medical organization and the attribute "Researcher" issued by the administrators of a clinical trial. On a commercial application, two corporations such as Boeing and General Electric might both issue attributes as part of a joint project. Using current ABE systems for these applications can be problematic since one needs a single authority that is both able to verify attributes across different organizations and issue private keys to every user in the system.

A Simple Approach and Its Limitations. We would like to realize an encryption system where a party can encrypt data for a policy written over attributes issued by different authorities. A user in the system should be able to decrypt if their attributes (possibly issued by multiple authorities) satisfy the policy specified by the ciphertext. In addition, the system should be able to express complex policies and not require coordination amongst the authorities. 
An initial step towards this goal is to simply "engineer" a system by utilizing existing (Ciphertext-Policy) Attribute-Based Encryption schemes along with standard signature schemes. In this proposal, a designated "central authority" will first create a set of public parameters. Then any party wishing to become an "authority" will create a signature verification key VK that will be associated with them. A user in the system with a globally verifiable identifier GID will collect private keys for attributes that it has from different authorities.

Suppose that a user GID can demonstrate attributes $X_{1}, X_{2}$ to the authority with verification key $\mathrm{VK}$ and attribute $Y$ to the authority with verification key $\mathrm{VK}^{\prime}$. The user will obtain his secret key as follows. First, he will obtain a signature of GID, $\left(X_{1}, X_{2}\right)$ that verifies under VK and a signature of GID, $Y$ under $\mathrm{VK}^{\prime}$ from the two respective authorities (and any other authorities). Next, the user will present these signature and verification key pairs to the central authority. The central authority will first check that each signature verifies under the claimed verification key and that each signature is on the same global identifier. Using an existing ABE algorithm, it will then issue an attribute for each verification key and attribute pair. In the above example, the user will get a key with attributes "VK, $X_{1}$ ", "VK, $X_{2}$ ", and "VK', $Y$ ". We note that the operation of the central authority is agnostic to the meaning of these verification keys and attributes; indeed, it will not need to have any a priori relationship with any of the authorities.

This simple system enjoys multiple benefits. Since encryption simply uses a prior ABE system, we can achieve the same level of expressiveness and write a policy in terms of any boolean formula. The system also requires minimum coordination between separate authorities. Any party can choose to be an authority by creating and publishing a verification key coupled with a list of attributes it will manage. Different authorities will not need to coordinate or even be aware of each other. There are several issues that will need to be dealt with in any larger system, such as the choice of an appropriate global identifier 1 or a party's decision as to which authority it trusts to issue private keys related to certain attributes. For instance, one might encrypt a policy using Experian's verification key to attest for the attribute of a good FICO (credit) score.

The major drawback of this simple engineered approach is that it requires a designated central authority. This authority must be globally trustworthy, since its failure will compromise the entire system. If we aim to build a large or even global scale system, this authority will become a common bottleneck. Spreading a central authority's keys over several machines to alleviate performance pressures might simultaneously increase the risk of key exposure.

A few works have attempted to create new cryptographic solutions to the multi-authority ABE problem. Chase 21] proposed an interesting solution that introduced the concept of using a global identifier as a "linchpin" for tying

${ }^{1}$ The idea of applying a global identifier in the context of multi-authority ABE was first proposed by Chase 21. Chase adapted the concept from its use in anonymous credential systems [19. One previously suggested candidate for a global identifier is a user's social security number. 
users' keys together. Her system relied on a central authority and was limited to expressing a strict "AND" policy over a pre-determined set of authorities. Therefore a party encrypting would be much more limited than in the simple engineering approach outlined above. Müller, Katzenbeisser, and Eckert [4243] give a different system with a centralized authority that realizes any LSSS access structure. Their construction builds on the Waters system [54; their proof is limited to non-adaptive queries. The system achieves roughly the same functionality as the engineering approach above, except one can still acquire attributes from additional authorities without revisiting the central authority. Chase and Chow [22] showed how to remove the central authority using a distributed PRF; however, the same limitations of an AND policy of a determined set of authorities remained. Lin et. al. 40, give a threshold based scheme that is also somewhat decentralized. The set of authorities is fixed ahead of time, and they must interact during the system setup. The system is only secure up to collusions of $m$ users, where $m$ is a system parameter chosen at setup such that the cost of operations and key storage scales with $m$.

Our Contribution. We propose a new multi-authority Attribute-Based Encryption system. In our system, any party can become an authority and there is no requirement for any global coordination other than the creation of an initial set of common reference parameters. (These will be created during a trusted setup.) A party can simply act as an authority by creating a public key and issuing private keys to different users that reflect their attributes. Different authorities need not even be aware of each other. We use the Chase 21. concept of global identifiers to "link" private keys together that were issued to the same user by different authorities. A user can encrypt data in terms of any boolean formula 2 over attributes issued from any chosen set of authorities.

Finally, our system does not require any central authority. We thus avoid the performance bottleneck incurred by relying on a central authority, which makes our system more scalable. We also avoid placing absolute trust in a single designated entity which must remain active and uncorrupted throughout the lifetime of the system. This is a crucial improvement for efficiency as well as security, since even a central authority that remains uncorrupted may occasionally fail for benign reasons, and a system that constantly relies on its participation will be forced to remain stagnant until it can be restored. In our system, authorities can function entirely independently, and the failure or corruption of some authorities will not affect the operation of functioning, uncorrupted authorities. This makes our system more robust then the other approaches outlined above.

Challenges and Our Techniques. In constructing our system, our central technical hurdle is to make it collusion resistant. Prior Attribute-Based Encryption systems achieved collusion resistance when the ABE system authority "tied" together different components (representing different attributes) of a user's private key by randomizing the key. Such randomization would make the different key

${ }^{2}$ Our system actually generalizes to handle any policy that can be expressed as a Linear Secret Sharing Scheme (LSSS) or equivalently a monotone span program. 
components compatible with each other, but not with the parts of a key issued to another user.

In our setting, we want to satisfy the simultaneous goals of autonomous key generation and collusion resistance. The requirement of autonomous key generation means that established techniques for key randomization cannot be applied since there is no one party to compile all the pieces together. Furthermore, in our system each component may come from a different authority, where such authorities have no coordination and are possibly not even aware of each other and there is no preset access structure 3 .

To overcome this, we develop a novel technique for tying a user's key components together and preventing collusion attacks between users with different global identifiers. At a high level, instead of relying on one key generation call to tie all key components together, we will use a hash function on the user's global identity, GID to manage collusion resistance across multiple key generations issued by different authorities.

In our system, we define a hash function $H$ (modeled as a random oracle) that hashes each identity to a (bilinear) group element. We will use the group element output from the hash function $H$ (GID) as the linchpin to tie keys together. Tying keys together in this manner is more challenging than in the single authority case. Our main idea is to structure the decryption mechanism at each satisfied node ' $x$ ' in the access tree such that a user will recover a target group element of the form $e(g, g)^{\lambda_{x}} \cdot e(g, H(\mathrm{GID}))^{w_{x}}$. This group element first contains a secret share $\lambda_{x}$ of a secret $s$ in the exponent, and these shares can be combined to recover the message. However, these will each be "blinded" by a share $w_{x}$ which is a share of 0 in the exponent with base $e(g, H(\mathrm{GID}))$. This structure allows for the decryption algorithm to both reconstruct the main secret and to unblind it in parallel. If a user with a particular identifier GID satisfies the access tree, he can reconstruct $s$ in the exponent by raising the group elements to the proper exponents. However, this operation will simultaneously reconstruct the share of 0 and thus the $e(g, H(\mathrm{GID}))$ terms will cancel out. Intuitively, if two users with different global identifiers GID, GID' attempt to collude, the cancelation will not work since the $w_{x}$ shares will have different bases.

We prove our system secure using the recent dual system encryption methodology [53], where the security proof works by first converting the challenge ciphertexts and private keys to a semi-functional form and then arguing security. We follow a recent variant of the dual system proof technique due to Lewko and Waters [39] and build our system using bilinear groups of composite order. The absence of coordination between the authorities also introduces a new technical challenge in applying the dual system encryption methodology. Due to the decentralized nature of user's keys, the techniques employed in [37] to achieve full security for single authority ABE using dual system encryption are insufficient. We overcome this by using two semi-functional subgroups instead of one, and switching between these allows us to defeat the information-theoretic problem

${ }^{3}$ Prior works 21 22 assumed coordination ahead of time between different authorities and required a limited access structure. 
which is naturally encountered if one simply tries to apply the previous techniques. We prove security under similar assumptions to the LW paper in the random oracle model.

Related Work. Several of the roots of Attribute-Based Encryption can be traced back to Identity Based Encryption (IBE), proposed by Shamir 46. The first IBE schemes were constructed by Boneh and Franklin [13] and Cocks [24]. These initial systems were proven secure in the random oracle model. Other standard model solutions followed [2010/52 27], along with extensions to the hierarchical IBE setting 342811 .

Attribute-based encryption was introduced by Sahai and Waters 45. Subsequently, Goyal, Pandey, Sahai, and Waters 30 formulated two complimentary forms of ABE: Ciphertext-Policy Attribute-Based Encryption (CP-ABE) and Key-Policy Attribute-Based Encryption (KP-ABE). In a CP-ABE system, keys are associated with sets of attributes and ciphertexts are associated with access policies. In a KP-ABE system, the situation is reversed: keys are associated with access policies and ciphertexts are associated with sets of attributes. Since then, several different ABE systems have been proposed [8/21 23 29 44 54 22], as well as related systems 142 . The problem of building ABE systems with multiple authorities was proposed by Sahai and Waters and first considered by Chase [21] and Chase and Chow 22]. Another interesting direction is the construction of "anonymous" or predicate encryption systems [36/49 17/12 144737] where in addition to the data the encryption policy or other properties are hidden. Other works have discussed similar problems without addressing collusion resistance 34515 184151. In these systems, the data encryptor specifies an access policy such that a set of users can decrypt the data only if the union of their credentials satisfies the access policy.

Until recently, all ABE systems were proven secure in the selective model where an attacker needed to declare the structure of the challenge ciphertext before seeing the public parameters. Recently, Lewko, Okamoto, Sahai, Takashima and Waters 37] solved the open problem by giving the first fully secure AttributeBased Encryption systems. Their system applied the dual system encryption methodology introduced by Waters [53] and techniques used by Lewko and Waters [39]. Our proof uses some techniques from Lewko et. al. [37, but faces new challenges from the multi-authority setting.

Organization. In Section 2, we formally define multi-authority CP-ABE systems and their security. In Section 3, we give our complexity assumptions. In Section 4, we present our multi-authority $\mathrm{CP}-\mathrm{ABE}$ system and outline the proof of its security. In Section 5, we discuss possible extensions of our results.

\section{Multi-authority CP-ABE}

Here we give the necessary background on multi-authority CP-ABE schemes and their security definition. For background on access structures, linear secret-sharing schemes, and composite order bilinear groups, see the full version of this paper [38]. 
A multi-authority Ciphertext-Policy Attribute-Based Encryption system is comprised of the following five algorithms:

Global $\operatorname{Setup}(\lambda) \rightarrow$ GP. The global setup algorithm takes in the security parameter $\lambda$ and outputs global parameters GP for the system.

Authority Setup(GP) $\rightarrow$ SK, PK. Each authority runs the authority setup algorithm with GP as input to produce its own secret key and public key pair, SK, PK.

$\operatorname{Encrypt}(M,(A, \rho), \mathrm{GP},\{\mathrm{PK}\}) \rightarrow \mathrm{CT}$. The encryption algorithm takes in a message $M$, an access matrix $(A, \rho)$, the set of public keys for relevant authorities, and the global parameters. It outputs a ciphertext CT.

KeyGen(GID, GP, $i, \mathrm{SK}) \rightarrow \mathrm{K}_{i, \mathrm{GID}}$. The key generation algorithm takes in an identity GID, the global parameters, an attribute $i$ belonging to some authority, and the secret key SK for this authority. It produces a key $\mathrm{K}_{i \text {,GID }}$ for this attribute, identity pair.

Decrypt $\left(\mathrm{CT}, \mathrm{GP},\left\{\mathrm{K}_{i, \mathrm{GID}}\right\}\right) \rightarrow M$. The decryption algorithm takes in the global parameters, the ciphertext, and a collection of keys corresponding to attribute, identity pairs all with the same fixed identity GID. It outputs either the message $M$ when the collection of attributes $i$ satisfies the access matrix corresponding to the ciphertext. Otherwise, decryption fails.

Definition 1. A multi-authority $C P-A B E$ system is said to be correct if whenever $\mathrm{GP}$ is obtained from the global setup algorithm, CT is obtained from the encryption algorithm on the message $M$, and $\left\{\mathrm{K}_{i, \mathrm{GID}}\right\}$ is a set of keys obtained from the key generation algorithm for the same identity GID and for a set of attributes satisfying the access structure of the ciphertext, Decrypt $\left(\mathrm{CT}, \mathrm{GP},\left\{\mathrm{K}_{i, \mathrm{GID}}\right\}\right)=M$.

\subsection{Security Definition}

We define security for multi-authority Ciphertext-Policy Attribute-Based Encryption systems by the following game between a challenger and an attacker. We assume that adversaries can corrupt authorities only statically, but key queries are made adaptively. A static corruption model is also used by Chase 21 and Chase and Chow 22, but we note that our model additionally allows the adversary to choose the public keys of the corrupted authorities for itself, instead of having these initially generated by the challenger.

We let $S$ denote the set of authorities and $U$ denote the universe of attributes. We assume each attribute is assigned to one authority (though each authority may control multiple attributes). In practice, we can think of an attribute as being the concatenation of an authority's public key and a string attribute. This will ensure that if multiple authorities choose the same string attribute, these will still correspond to distinct attributes in the system. 
Setup. The global setup algorithm is run. The attacker specifies a set $S^{\prime} \subseteq S$ of corrupt authorities. For good (non-corrupt) authorities in $S-S^{\prime}$, the challenger obtains public key, private key pairs by running the authority setup algorithm, and gives the public keys to the attacker.

Key Query Phase 1. The attacker makes key queries by submitting pairs ( $i$, GID) to the challenger, where $i$ is an attribute belonging to a good authority and GID is an identity. The challenger responds by giving the attacker the corresponding key, $\mathrm{K}_{i, \mathrm{GID}}$.

Challenge Phase. The attacker must specify two messages, $M_{0}, M_{1}$, and an access matrix $(A, \rho)$. The access matrix must satisfy the following constraint. We let $V$ denote the subset of rows of $A$ labeled by attributes controlled by corrupt authorities. For each identity GID, we let $V_{\mathrm{GID}}$ denote the subset of rows of $A$ labeled by attributes $i$ for which the attacker has queried $(i$, GID). For each GID, we require that the subspace spanned by $V \cup V_{\mathrm{GID}}$ must not include $(1,0, \ldots, 0)$. (In other words, the attacker cannot ask for a set of keys that allow decryption, in combination with any keys that can obtained from corrupt authorities.) The attacker must also give the challenger the public keys for any corrupt authorities whose attributes appear in the labeling $\rho$. The challenger flips a random coin $\beta \in\{0,1\}$ and sends the attacker an encryption of $M_{\beta}$ under access matrix $(A, \rho)$.

Key Query Phase 2. The attacker may submit additional key queries ( $i$, GID), as long as they do not violate the constraint on the challenge matrix $(A, \rho)$.

Guess. The attacker must submit a guess $\beta^{\prime}$ for $\beta$. The attacker wins if $\beta=\beta^{\prime}$. The attacker's advantage in this game is defined to be $\operatorname{Pr}\left[\beta=\beta^{\prime}\right]-\frac{1}{2}$.

Definition 2. A multi-authority Ciphertext-Policy Attribute-Based Encryption system is secure (against static corruption of authorities) if all polynomial time attackers have at most a negligible advantage in this security game.

\subsection{Transformation from One-Use Multi-authority CP-ABE}

In the full version of this paper, we show how to construct a fully secure multiauthority CP-ABE system where attributes are used multiple times in an access matrix from a fully secure multi-authority CP-ABE system where attributes are used only once. We do this with a simple encoding technique. This same transformation was employed by 37] for (single authority) CP-ABE.

\section{Our Assumptions}

We now state the complexity assumptions that we will rely on to prove security for our system. These assumptions are formulated for a bilinear group $G$ of order $N=p_{1} p_{2} p_{3}$, a product of 3 primes. We let $e: G \times G \rightarrow G_{T}$ denote the bilinear 
map. For background on these groups, see the full version. We note that these are similar to the assumptions used in [3937]. While the fourth assumption is new, the first three are instances of the class of General Subgroup Decision Assumptions described in [7. This class is defined as follows: in a bilinear group of order $N=p_{1} p_{2} \ldots p_{n}$, there is a subgroup of order $\prod_{i \in S} p_{i}$ for each subset $S \subseteq\{1, \ldots, n\}$. We let $S_{0}, S_{1}$ denote two distinct subsets. We then assume it is hard to distinguish a random element from the subgroup associated with $S_{0}$ from a random element of the subgroup associated with $S_{1}$, even if one is given random elements from subgroups associated with several subsets $Z_{i}$ which each satisfy either that $S_{0} \cap Z_{i}=\emptyset=S_{1} \cap Z_{i}$ or $S_{0} \cap Z_{i} \neq \emptyset \neq S_{1} \cap Z_{i}$. We prove our four specific assumptions are generically secure in the full version, under the assumption that it is hard to find a nontrivial factor of the group order $N$.

In the assumptions below, we let $G_{p_{1}}$, e.g., denote the subgroup of order $p_{1}$ in $G$. We note that if $g_{i} \in G_{p_{i}}$ and $g_{j} \in G_{p_{j}}$ for $i \neq j$, then $e\left(g_{i}, g_{j}\right)=1$. When we write $g_{1} \stackrel{R}{\longleftarrow} G_{p_{1}}$, we mean that $g_{1}$ is chosen to be a random generator of $G_{p_{1}}$ (so it is not the identity element). Similarly, when we write $T_{1} \stackrel{R}{\longleftarrow} G$, we mean that $T_{1}$ is chosen to be a random generator of $G$ (this is not quite the same as a uniformly random element, but the distributions are negligibly close).

Assumption 1 (Subgroup decision problem for 3 primes). Given a group generator $\mathcal{G}$, we define the following distribution:

$$
\begin{gathered}
\mathbb{G}=\left(N=p_{1} p_{2} p_{3}, G, G_{T}, e\right) \stackrel{R}{\longleftarrow} \mathcal{G}, \\
g_{1} \stackrel{R}{\longleftarrow} G_{p_{1}}, \\
D=\left(\mathbb{G}, g_{1}\right), \\
T_{1} \stackrel{R}{\longleftarrow} G, T_{2} \stackrel{R}{\longleftarrow} G_{p_{1}} .
\end{gathered}
$$

We define the advantage of an algorithm $\mathcal{A}$ in breaking Assumption 1 to be:

$$
A d v 1_{\mathcal{G}, \mathcal{A}}(\lambda):=\left|\operatorname{Pr}\left[\mathcal{A}\left(D, T_{1}\right)=1\right]-\operatorname{Pr}\left[\mathcal{A}\left(D, T_{2}\right)=1\right]\right| .
$$

We note that $T_{1}$ can be written (uniquely) as the product of an element of $G_{p_{1}}$, an element of $G_{p_{2}}$, and an element of $G_{p_{3}}$. We refer to these elements as the " $G_{p_{1}}$ part of $T_{1}$ ", the " $G_{p_{2}}$ part of $T_{1}$ ", and the " $G_{p_{3}}$ part of $T_{1}$ " respectively. We will use this terminology in our proofs.

Definition 3. We say that $\mathcal{G}$ satisfies Assumption 1 if $A d v 1_{\mathcal{G}, \mathcal{A}}(\lambda)$ is a negligible function of $\lambda$ for any polynomial time algorithm $\mathcal{A}$.

Assumption 2. Given a group generator $\mathcal{G}$, we define the following distribution:

$$
\begin{gathered}
\mathbb{G}=\left(N=p_{1} p_{2} p_{3}, G, G_{T}, e\right) \stackrel{R}{\longleftarrow} \mathcal{G}, \\
g_{1}, X_{1} \stackrel{R}{\longleftarrow} G_{p_{1}}, X_{2} \stackrel{R}{\longleftarrow} G_{p_{2}}, g_{3} \stackrel{R}{\longleftarrow} G_{p_{3}},
\end{gathered}
$$




$$
\begin{gathered}
D=\left(\mathbb{G}, g_{1}, g_{3}, X_{1} X_{2}\right), \\
T_{1} \stackrel{R}{\stackrel{R}{\longleftarrow}} G_{p_{1}}, T_{2} \stackrel{R}{\longleftarrow} G_{p_{1} p_{2}} .
\end{gathered}
$$

We define the advantage of an algorithm $\mathcal{A}$ in breaking Assumption 2 to be:

$$
A d v 2_{\mathcal{G}, \mathcal{A}}(\lambda):=\left|\operatorname{Pr}\left[\mathcal{A}\left(D, T_{1}\right)=1\right]-\operatorname{Pr}\left[\mathcal{A}\left(D, T_{2}\right)=1\right]\right|
$$

Definition 4. We say that $\mathcal{G}$ satisfies Assumption 2 if $A d v 2_{\mathcal{G}, \mathcal{A}}(\lambda)$ is a negligible function of $\lambda$ for any polynomial time algorithm $\mathcal{A}$.

Assumption 3. Given a group generator $\mathcal{G}$, we define the following distribution:

$$
\begin{gathered}
\mathbb{G}=\left(N=p_{1} p_{2} p_{3}, G, G_{T}, e\right), \stackrel{R}{\longleftarrow} \mathcal{G}, \\
g_{1}, X_{1} \stackrel{R}{\longleftarrow} G_{p_{1}}, Y_{2} \stackrel{R}{\longleftarrow} G_{p_{2}}, X_{3}, Y_{3} \stackrel{R}{\longleftarrow} G_{p_{3}}, \\
D=\left(\mathbb{G}, g_{1}, X_{1} X_{3}, Y_{2} Y_{3}\right), \\
T_{1} \stackrel{R}{\longleftarrow} G_{p_{1} p_{2}}, T_{2} \stackrel{R}{\longleftarrow} G_{p_{1} p_{3}} .
\end{gathered}
$$

We define the advantage of an algorithm $\mathcal{A}$ in breaking Assumption 3 to be:

$$
A d v 3_{\mathcal{G}, \mathcal{A}}(\lambda):=\left|\operatorname{Pr}\left[\mathcal{A}\left(D, T_{1}\right)=1\right]-\operatorname{Pr}\left[\mathcal{A}\left(D, T_{2}\right)=1\right]\right|
$$

Definition 5. We say that $\mathcal{G}$ satisfies Assumption 3 if $A d v 3_{\mathcal{G}, \mathcal{A}}(\lambda)$ is a negligible function of $\lambda$ for any polynomial time algorithm $\mathcal{A}$.

Assumption 4. Given a group generator $\mathcal{G}$, we define the following distribution:

$$
\begin{gathered}
\mathbb{G}=\left(N=p_{1} p_{2} p_{3}, G, G_{T}, e\right), \stackrel{R}{\longleftarrow} \mathcal{G}, \\
g_{1} \stackrel{R}{\longleftarrow} G_{p_{1}}, g_{2} \stackrel{R}{\longleftarrow} G_{p_{2}}, g_{3} \stackrel{R}{\longleftarrow} G_{p_{3}}, a, b, c, d \stackrel{R}{\longleftarrow} \mathbb{Z}_{N}, \\
D=\left(\mathbb{G}, g_{1}, g_{2}, g_{3}, g_{1}^{a}, g_{1}^{b} g_{3}^{b}, g_{1}^{c}, g_{1}^{a c} g_{3}^{d}\right), \\
T_{1}=e\left(g_{1}, g_{1}\right)^{a b c}, T_{2} \stackrel{R}{\longleftarrow} G_{T} .
\end{gathered}
$$

We define the advantage of an algorithm $\mathcal{A}$ in breaking Assumption 4 to be:

$$
A d v 4_{\mathcal{G}, \mathcal{A}}(\lambda):=\left|\operatorname{Pr}\left[\mathcal{A}\left(D, T_{1}\right)=1\right]-\operatorname{Pr}\left[\mathcal{A}\left(D, T_{2}\right)=1\right]\right|
$$

Definition 6. We say that $\mathcal{G}$ satisfies Assumption 4 if $A d v 4_{\mathcal{G}, \mathcal{A}}(\lambda)$ is a negligible function of $\lambda$ for any polynomial time algorithm $\mathcal{A}$. 


\section{Our Multi-authority CP-ABE System}

We now present our one-use multi-authority ciphertext-policy attribute based encryption system. We use a composite order bilinear group $G$, where the group order is a product of three primes: $N=p_{1} p_{2} p_{3}$. Except for the random oracle $H$ which maps identities to random group elements, the entire system is confined to the subgroup $G_{p_{1}}$ in $G$. The subgroups $G_{p_{2}}$ and $G_{p_{3}}$ are used in our security proof, which employs the dual system encryption technique. In a dual system, keys and ciphertexts can be either normal or semi-functional. Normal keys and ciphertexts in our system will be contained in the subgroup $G_{p_{1}}$, while semifunctional keys and ciphertexts will involve elements of the subgroups $G_{p_{2}}$ and $G_{p_{3}}$. In other words, the subgroups $G_{p_{2}}$ and $G_{p_{3}}$ form the semi-functional space, which is orthogonal to the subgroup $G_{p_{1}}$ where the normal keys and ciphertexts reside.

To prevent collusion attacks, our system uses the global identity to "tie" together the various attributes belonging to a specific user so that they cannot be successfully combined with another's user's attributes in decryption. More specifically, the encryption algorithm blinds the message $M$ with $e\left(g_{1}, g_{1}\right)^{s}$, where $g_{1}$ is a generator of the subgroup $G_{p_{1}}$, and $s$ is a randomly chosen value in $\mathbb{Z}_{N}$. The value $s$ is then split into shares $\lambda_{x}$ according to the LSSS matrix, and the value 0 is split into shares $\omega_{x}$. The decryptor must recover the blinding factor $e\left(g_{1}, g_{1}\right)^{s}$ by pairing their keys for attribute, identity pairs ( $i$, GID) with the ciphertext elements to obtain the shares of $s$. In doing so, the decryptor will introduce terms of the form $e\left(g_{1}, H(\mathrm{GID})\right)^{\omega_{x}}$. If the decryptor has a satisfying set of keys with the same identity GID, these additional terms will cancel from the final result, since the $\omega_{x}$ 's are shares of 0 . If two users with different identities GID and GID' attempt to collude and combine their keys, then there will be some terms of the form $e\left(g_{1}, H(\mathrm{GID})\right)^{\omega_{x}}$ and some terms of the form $e\left(g_{1}, H\left(\mathrm{GID}^{\prime}\right)\right)^{\omega_{x^{\prime}}}$, and these will not cancel with each other, thereby preventing the recovery of $e\left(g_{1}, g_{1}\right)^{s}$.

\subsection{Construction}

Global $\operatorname{Setup}(\lambda) \rightarrow$ GP. In the global setup, a bilinear group $G$ of order $N=$ $p_{1} p_{2} p_{3}$ is chosen. The global public parameters, GP, are $N$ and a generator $g_{1}$ of $G_{p_{1}}$. In addition, the description of a hash function $H:\{0,1\}^{*} \rightarrow G$ that maps global identities GID to elements of $G$ is published. We will model $H$ as a random oracle.

Authority Setup $(\mathrm{GP}) \rightarrow \mathrm{PK}, \mathrm{SK}$. For each attribute $i$ belonging to the authority, the authority chooses two random exponents $\alpha_{i}, y_{i} \in \mathbb{Z}_{N}$ and publishes $\mathrm{PK}_{j}=$ $\left\{e\left(g_{1}, g_{1}\right)^{\alpha_{i}}, g_{1}^{y_{i}} \forall i\right\}$ as its public key. It keeps $\mathrm{SK}=\left\{\alpha_{i}, y_{i} \forall i\right\}$ as its secret key.

$\operatorname{Encrypt}(M,(A, \rho), \mathrm{GP},\{\mathrm{PK}\}) \rightarrow \mathrm{CT}$. The encryption algorithm takes in a message $M$, an $n \times \ell$ access matrix $A$ with $\rho$ mapping its rows to attributes, the global parameters, and the public keys of the relevant authorities. It chooses a random $s \in \mathbb{Z}_{N}$ and a random vector $v \in \mathbb{Z}_{N}^{\ell}$ with $s$ as its first entry. We let $\lambda_{x}$ 
denote $A_{x} \cdot v$, where $A_{x}$ is row $x$ of $A$. It also chooses a random vector $w \in \mathbb{Z}_{N}^{\ell}$ with 0 as its first entry. We let $\omega_{x}$ denote $A_{x} \cdot w$. For each row $A_{x}$ of $A$, it chooses a random $r_{x} \in \mathbb{Z}_{N}$. The ciphertext is computed as:

$$
\begin{gathered}
C_{0}=M e\left(g_{1}, g_{1}\right)^{s}, C_{1, x}=e\left(g_{1}, g_{1}\right)^{\lambda_{x}} e\left(g_{1}, g_{1}\right)^{\alpha_{\rho(x)} r_{x}}, \\
C_{2, x}=g_{1}^{r_{x}}, C_{3, x}=g_{1}^{y_{\rho(x)} r_{x}} g_{1}^{\omega_{x}} \forall x .
\end{gathered}
$$

KeyGen(GID, $i, \mathrm{SK}, \mathrm{GP}) \rightarrow \mathrm{K}_{i, \mathrm{GID}}$. To create a key for GID for attribute $i$ belonging to an authority, the authority computes:

$$
\mathrm{K}_{i, \mathrm{GID}}=g_{1}^{\alpha_{i}} H(\mathrm{GID})^{y_{i}} .
$$

$\operatorname{Decrypt}\left(\mathrm{CT},\left\{\mathrm{K}_{i, \mathrm{GID}}\right\}, \mathrm{GP}\right) \rightarrow M$. We assume the ciphertext is encrypted under an access matrix $(A, \rho)$. To decrypt, the decryptor first obtains $H$ (GID) from the random oracle. If the decryptor has the secret keys $\left\{\mathrm{K}_{\rho(x) \text {,GID }}\right\}$ for a subset of rows $A_{x}$ of $A$ such that $(1,0, \ldots, 0)$ is in the span of these rows, then the decryptor proceeds as follows. For each such $x$, the decryptor computes:

$$
C_{1, x} \cdot e\left(H(\mathrm{GID}), C_{3, x}\right) / e\left(\mathrm{~K}_{\rho(x), \mathrm{GID}}, C_{2, x}\right)=e\left(g_{1}, g_{1}\right)^{\lambda_{x}} e\left(H(\mathrm{GID}), g_{1}\right)^{\omega_{x}} .
$$

The decryptor then chooses constants $c_{x} \in \mathbb{Z}_{N}$ such that

$$
\begin{aligned}
\sum_{x} c_{x} A_{x}= & (1,0, \ldots, 0) \text { and computes: } \\
& \prod_{x}\left(e\left(g_{1}, g_{1}\right)^{\lambda_{x}} e\left(H(\mathrm{GID}), g_{1}\right)^{\omega_{x}}\right)^{c_{x}}=e\left(g_{1}, g_{1}\right)^{s} .
\end{aligned}
$$

(We recall that $\lambda_{x}=A_{x} \cdot v$ and $\omega_{x}=A_{x} \cdot w$, where $v \cdot(1,0, \ldots, 0)=s$ and $w \cdot(1,0, \ldots, 0)=0$.) The message can then be obtained as:

$$
M=C_{0} / e\left(g_{1}, g_{1}\right)^{s} .
$$

\subsection{Security}

We apply a form of the dual system encryption technique to prove security; overcoming the new challenges that arise in the multi-authority setting. In a dual system, keys and ciphertexts can either be normal or semi-functional: normal keys can decrypt semi-functional ciphertexts, semi-functional keys can decrypt normal ciphertexts, but semi-functional keys cannot decrypt semi-functional ciphertexts. The proof proceeds by a hybrid argument over a sequence of games, where we first change the challenge ciphertext to be semi-functional, and then change the keys to be semi-functional one by one. To prove that these games are indistinguishable, we must ensure that the simulator cannot test the form of the key being turned from normal to semi-functional for itself by test decrypting a semi-functional ciphertext. We avoid this problem employing the approach of [3937, where the simulator can only make a challenge ciphertext and key pair which is nominally semi-functional, meaning that both the key and ciphertext have semi-functional components, but these cancel out upon decryption. Thus, if the simulator attempts to test the form of the key for itself, decryption will succeed unconditionally. 
New Challenges. The existence of multiple authorities who do not coordinate with each other introduces additional technical challenges in our case. Nominal semi-functionality must be hidden from the attacker's view, which is accomplished in [37] by using temporary "blinding factors" in the semi-functional space that are active for one key at a time. Leaving these blinding factors off for the other keys prevents leakage of information that would informationtheoretically reveal nominal semi-functionality in the attacker's view. However, what allows these blinding factors to be turned on and off is the stable presence of a semi-functional term attached to a single element in each key derived from the master secret key. In the multi-authority case, we do not have this sort of structural linchpin to rely on. We still need the blinding factors to hide nominal semi-functionality, but we cannot simply excise them from the other semi-functional keys to prevent their leakage. To overcome this, we use two subgroups for the semi-functional space, and instead of removing the blinding factors from the other keys, we "switch" them from one semi-functional subgroup to the other. This switch preserves semi-functionality of the keys while avoiding leakage of information about the subgroup the semi-functional components have been switched out of.

Hybrid Organization. We now formally define our sequence of games. We will assume a one-use restriction on attributes throughout the proof: this means that the row labeling $\rho$ of the challenge ciphertext access matrix $(A, \rho)$ must be injective.

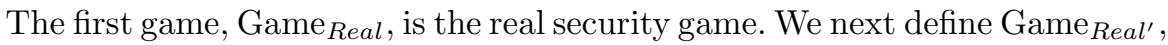
which is like the real security game, except that the random oracle maps identities GID to random elements of $G_{p_{1}}$ instead of $G$. We now define semi-functional ciphertexts and keys, which are used only in the proof - not in the real system.

Semi-functional ciphertexts will contain terms from subgroups $G_{p_{2}}$ and $G_{p_{3}}$. Semi-functional keys will be of two types: semi-functional keys of Type 1 will have terms in $G_{p_{2}}$, while semi-functional keys of Type 2 will have terms in $G_{p_{3}}$. When a semi-functional key of Type 1 is used to decrypt a semi-functional ciphertext, the extra terms from $G_{p_{2}}$ in the key will be paired with the extra $G_{p_{2}}$ terms in the ciphertext, which will cause decryption to fail. When a semi-functional key of Type 2 is used to decrypt a semi-functional ciphertext, the extra terms from $G_{p_{3}}$ in the key will be paired with the extra $G_{p_{3}}$ terms in the ciphertext, which will cause decryption to fail.

To more precisely describe semi-functional ciphertexts and keys, we first fix random values $z_{i}, t_{i} \in \mathbb{Z}_{N}$ for each attribute $i$ which will be common to semifunctional ciphertexts and keys. These values are fixed per attribute, and do not vary for different users.

Semi-functional Ciphertexts. To create a semi-functional ciphertext, we first run the encryption algorithm to obtain a normal ciphertext,

$$
C_{0}^{\prime}, C_{1, x}^{\prime}, C_{2, x}^{\prime}, C_{3, x}^{\prime} \forall x
$$


We let $g_{2}, g_{3}$ denote generators of $G_{p_{2}}$ and $G_{p_{3}}$ respectively. We choose two random vectors $u_{2}, u_{3} \in \mathbb{Z}_{N}^{\ell}$ and set $\delta_{x}=A_{x} \cdot u_{2}, \sigma_{x}=A_{x} \cdot u_{3}$ for each row $A_{x}$ of the access matrix $A$. We let $B$ denote the subset of rows of $A$ whose corresponding attributes belong to corrupted authorities. We let $\bar{B}$ be the subset of rows of $A$ whose corresponding attributes belong to good authorities. For each row $A_{x} \in \bar{B}$, we also choose random exponents $\gamma_{x}, \psi_{x} \in \mathbb{Z}_{N}$. The semi-functional ciphertext is formed as:

$$
\begin{aligned}
& C_{0}=C_{0}^{\prime}, C_{1, x}=C_{1, x}^{\prime}, C_{2, x}=C_{2, x}^{\prime} g_{2}^{\gamma_{x}} g_{3}^{\psi_{x}}, \\
& C_{3, x}=C_{3, x}^{\prime} g_{2}^{\delta_{x}+\gamma_{x} z_{\rho(x)}} g_{3}^{\sigma_{x}+\psi_{x} t_{\rho(x)}} \forall x \text { s.t. } A_{x} \in \bar{B}, \\
& C_{1, x}=C_{1, x}^{\prime}, C_{2, x}=C_{2, x}^{\prime}, C_{3, x}=C_{3, x}^{\prime} g_{2}^{\delta_{x}} g_{3}^{\sigma_{x}} \forall x \text { s.t. } A_{x} \in B .
\end{aligned}
$$

We say a ciphertext is nominally semi-functional when the values $\delta_{x}$ are shares of 0 .

Semi-functional Keys. We define the key for identity GID to be the collection of $H$ (GID) and all keys $\mathrm{K}_{i \text {,GID }}$ for attributes $i$ belonging to good authorities requested by the attacker throughout the game. (These queries may occur at different times.) Semi-functional keys for an identity GID will be of two types: Type 1 or Type 2 . To create a semi-functional key for identity GID, we let $H^{\prime}$ (GID) be a random element of $G_{p_{1}}$, and we choose a random exponent $c \in \mathbb{Z}_{N}$.

To create a semi-functional key of Type 1, we define the random oracle's output on GID to be:

$$
H(\mathrm{GID})=H^{\prime}(\mathrm{GID}) g_{2}^{c} \text {. }
$$

We create $\mathrm{K}_{i \text {,GID }}$ (for an attribute $i$ controlled by a good authority) by first creating a normal key $\mathrm{K}_{i, \mathrm{GID}}^{\prime}$ and setting:

$$
\mathrm{K}_{i, \mathrm{GID}}=\mathrm{K}_{i, \mathrm{GID}}^{\prime} g_{2}^{c z_{i}} .
$$

To create a semi-functional key of Type 2, we define the random oracle's output on GID to be:

$$
H(\mathrm{GID})=H^{\prime}(\mathrm{GID}) g_{3}^{c} .
$$

We create $\mathrm{K}_{i \text {,GID }}$ (for an attribute $i$ controlled by a good authority) by first creating a normal key $\mathrm{K}_{i, \mathrm{GID}}^{\prime}$ and setting:

$$
\mathrm{K}_{i, \mathrm{GID}}=\mathrm{K}_{i, \mathrm{GID}}^{\prime} g_{3}^{c t_{i}} .
$$

We note that when a semi-functional key of Type 1 is used to decrypt a semifunctional ciphertext, the additional terms $e\left(g_{2}, g_{2}\right)^{c \delta_{x}}$ prevent decryption from succeeding, except when the values $\delta_{x}$ are shares of 0 (i.e. when we have a nominally semi-functional ciphertext). When a semi-functional key of Type 2 is used to decrypt a semi-functional ciphertext, the additional terms $e\left(g_{3}, g_{3}\right)^{c \sigma_{x}}$ prevent successful decryption.

We now define Game $_{0}$, which is like Game Real $^{\prime}$, except that the ciphertext given to the attacker is semi-functional. We let $q$ be the number of identities GID for which the attacker makes key queries $\mathrm{K}_{i, \mathrm{GID}}$. We define $\mathrm{Game}_{j, 1}$ and Game $_{j, 2}$ for each $j$ from 1 to $q$ as follows: 
$G_{a m e}{ }_{j, 1}$. This is like Game 0 , except that for the first $j-1$ queried identities, the received keys are semi-functional of Type 2 , and the received key for the $j^{t h}$ queried identity is semi-functional of Type 1 . The remaining keys are normal.

$G_{a m e}, 2$. This is like Game 0 , except that for the first $j$ queried identities, the received keys are semi-functional of Type 2 . The remaining keys are normal. We note that in Game $q, 2$, all keys are semi-functional of Type 2.

Game $_{\text {Final }}$. In this game, all keys are semi-functional of Type 2, and the ciphertext is a semi-functional encryption of a random message. We note that the attacker has advantage 0 in this game.

We show these games are indistinguishable in the following lemmas. We give the most interesting proof below, and the remaining proofs can be found in the full version of this paper.

Lemma 1. Suppose there exists a polynomial time algorithm $\mathcal{A}$ such that Game $_{\text {Real }} A d v_{\mathcal{A}}-$ Game $_{\text {Real }} A d v_{\mathcal{A}}=\epsilon$. Then we can construct a polynomial time algorithm $\mathcal{B}$ with advantage $\epsilon$ in breaking Assumption 1.

Lemma 2. Suppose there exists a polynomial time algorithm $\mathcal{A}$ such that Game $_{\text {Real }} A d v_{\mathcal{A}}-G_{a m e} A d v_{\mathcal{A}}=\epsilon$. Then we can construct a polynomial time algorithm $\mathcal{B}$ with advantage negligibly close to $\epsilon$ in breaking Assumption 1.

Lemma 3. Suppose there exists a polynomial time algorithm $\mathcal{A}$ such that Game $_{j-1,2} A d v_{\mathcal{A}}-$ Game $_{j, 1} A d v_{\mathcal{A}}=\epsilon$. Then we can construct a polynomial time algorithm $\mathcal{B}$ with advantage negligibly close to $\epsilon$ in breaking Assumption 2.

Proof. $\mathcal{B}$ receives $g_{1}, g_{3}, X_{1} X_{2}, T . \mathcal{B}$ will simulate either Game $_{j-1,2}$ or Game $_{j, 1}$ with $\mathcal{A}$, depending on the value of $T$. $\mathcal{B}$ outputs $g_{1}$ as the public generator of $G_{p_{1}}$ and $N$ as the group order. $\mathcal{A}$ specifies a set $S^{\prime} \subseteq S$ of corrupt authorities, where $S$ is the the set of all authorities in the system. For each attribute $i$ belonging to a good authority, $\mathcal{B}$ chooses random exponents $\alpha_{i}, y_{i} \in \mathbb{Z}_{N}$ and gives $\mathcal{A}$ the public parameters $e\left(g_{1}, g_{1}\right)^{\alpha_{i}}, g_{1}^{y_{i}}$.

We let $\operatorname{GID}_{k}$ denote the $k^{t h}$ identity queried by $\mathcal{A}$. When $\mathcal{A}$ first queries the random oracle for $H\left(\mathrm{GID}_{k}\right)$, if $k>j$, then $\mathcal{B}$ chooses a random exponent $h_{\mathrm{GID}_{k}} \in \mathbb{Z}_{N}$ and sets $H\left(\mathrm{GID}_{k}\right)=g_{1}^{h_{\mathrm{GID}_{k}}}$. If $k<j$, then $\mathcal{B}$ chooses a random exponent $h_{\mathrm{GID}_{k}} \in \mathbb{Z}_{N}$ and sets $H\left(\mathrm{GID}_{k}\right)=\left(g_{1} g_{3}\right)^{h_{\mathrm{GID}_{k}}}$ (we note that this is a random element of $G_{p_{1} p_{3}}$ since the values of $h_{\mathrm{GID}_{k}}$ modulo $p_{1}$ and modulo $p_{3}$ are uncorrelated). When $k=j, \mathcal{B}$ chooses a random exponent $h_{\mathrm{GID}_{j}} \in \mathbb{Z}_{N}$ and sets $H\left(\mathrm{GID}_{j}\right)=T^{h_{\mathrm{GID}_{j}}}$. In all cases, it stores this value so that it can respond consistently if $H\left(\mathrm{GID}_{k}\right)$ is queried again.

When $\mathcal{A}$ makes a key query $\left(i, \mathrm{GID}_{k}\right), \mathcal{B}$ responds as follows. If $H\left(\mathrm{GID}_{k}\right)$ has already been fixed, then $\mathcal{B}$ retrieves the stored value. Otherwise, $\mathcal{B}$ creates $H\left(\operatorname{GID}_{k}\right)$ according to $k$ as above. $\mathcal{B}$ forms the key as:

$$
\mathrm{K}_{i, \mathrm{GID}_{k}}=g_{1}^{\alpha_{i}} H\left(\mathrm{GID}_{k}\right)^{y_{i}}
$$


Notice that for $k<j, \mathcal{B}$ forms properly distributed semi-functional keys of Type 2 , where $t_{i}$ is congruent to $y_{i}$ modulo $p_{3}$ (these are uncorrelated from the values of $y_{i}$ modulo $p_{1}$ which appear in the public parameters). Also recall that the values $t_{i}$ are fixed per attribute, and do not vary across different keys. For $k>j, \mathcal{B}$ forms properly distributed normal keys. For $k=j, \mathcal{B}$ forms a normal key if $T \in G_{p_{1}}$ and a semi-functional key of Type 1 if $T \in G_{p_{1} p_{2}}$.

At some point, $\mathcal{A}$ gives $\mathcal{B}$ two messages, $M_{0}, M_{1}$, and an access matrix $(A, \rho)$. $\mathcal{B}$ flips a random coin $\beta \in\{0,1\}$, and encrypts $M_{\beta}$ as follows. (We note that $\mathcal{B}$ will produce a nominally semi-functional ciphertext, but this will be hidden from $\mathcal{A}$ 's view.) First, $\mathcal{B}$ chooses a random $s \in \mathbb{Z}_{N}$ and sets $C_{0}=M e\left(g_{1}, g_{1}\right)^{s}$. $\mathcal{B}$ also chooses three vectors, $v=\left(s, v_{2}, \ldots, v_{\ell}\right), w=\left(0, w_{2}, \ldots, w_{\ell}\right), u=\left(u_{1}, \ldots, u_{\ell}\right)$, where $v_{2}, \ldots, v_{\ell}, w_{2}, \ldots, w_{\ell}, u_{1}, \ldots, u_{\ell}$ are chosen randomly from $\mathbb{Z}_{N}$. We let $\lambda_{x}=A_{x} \cdot v, \omega_{x}=A_{x} \cdot w$, and $\sigma_{x}=A_{x} \cdot u$.

$\mathcal{A}$ additionally supplies $\mathcal{B}$ with public parameters $g^{y_{i}}, e\left(g_{1}, g_{1}\right)^{\alpha_{i}}$ for attributes $i$ belonging to corrupt authorities which are included in the access matrix $(A, \rho)$. We let $B$ denote the subset of rows of $A$ whose corresponding attributes belong to corrupted authorities. We let $\bar{B}$ be the subset of rows of $A$ whose corresponding attributes belong to good authorities. For each row $A_{x}$ in $B, \mathcal{B}$ chooses a random value $r_{x} \in \mathbb{Z}_{N}$. For each row $A_{x} \in \bar{B}, \mathcal{B}$ chooses random values $\psi_{x}, r_{x}^{\prime} \in \mathbb{Z}_{N}$, and will implicitly set $r_{x}=r r_{x}^{\prime}$, where $g_{1}^{r}$ is $X_{1}$.

For each row $A_{x} \in B$, the ciphertext is formed as:

$$
\begin{gathered}
C_{1, x}=e\left(g_{1}, g_{1}\right)^{\lambda_{x}}\left(e\left(g_{1}, g_{1}\right)^{\alpha_{\rho(x)}}\right)^{r_{x}}, \\
C_{2, x}=g_{1}^{r_{x}}, C_{3, x}=\left(g_{1}^{y_{\rho(x)}}\right)^{r_{x}}\left(X_{1} X_{2}\right)^{\omega_{x}} g_{3}^{\sigma_{x}} .
\end{gathered}
$$

For each row $A_{x} \in \bar{B}$, the ciphertext is formed as:

$$
\begin{gathered}
C_{1, x}=e\left(g_{1}, g_{1}\right)^{\lambda_{x}} e\left(g_{1}, X_{1} X_{2}\right)^{\alpha_{\rho(x)} r_{x}^{\prime}} \\
C_{2, x}=\left(X_{1} X_{2}\right)^{r_{x}^{\prime}} g_{3}^{\psi_{x}}, C_{3, x}=\left(X_{1} X_{2}\right)^{y_{\rho(x)} r_{x}^{\prime}} g_{3}^{y_{\rho(x)} \psi_{x}}\left(X_{1} X_{2}\right)^{\omega_{x}} g_{3}^{\sigma_{x}} .
\end{gathered}
$$

We note that the $X_{1}^{\omega_{x}}$ is $g_{1}^{A_{x} \cdot r w}$, and $r w$ is a random vector with first coordinate equal to 0 . This is a semi-functional ciphertext with parameters $\delta_{x}=A_{x} \cdot \mathrm{cw}$ modulo $p_{2}$ where $g_{2}^{c}$ is $X_{2}, g_{2}^{\gamma_{x}}$ equals $X_{2}^{r_{x}^{\prime}}, z_{\rho(x)}=y_{\rho(x)}$ modulo $p_{2}$, and $t_{\rho(x)}=$ $y_{\rho(x)}$ modulo $p_{3}$.

To see that this is properly distributed, we note that since $r_{x}^{\prime}, y_{\rho(x)}$ are chosen randomly in $\mathbb{Z}_{N}$, their values modulo $p_{1}$ and modulo $p_{2}$ are uncorrelated. This means that our $\gamma_{x}, \psi_{x}, z_{\rho(x)}, t_{\rho(x)}$ parameters are randomly distributed. It is clear that $\sigma_{x}$ is properly distributed, since it is a share of a random vector. The entries $w_{2}, \ldots, w_{\ell}$ of $w$ are also randomly distributed modulo $p_{2}$, however the $\delta_{x}$ 's are shares of 0 from the simulator's perspective. We must argue that these appear to be shares of a random exponent in $\mathcal{A}$ 's view.

We let the space $R$ denote the span of the rows of $A$ whose attributes are in $B$ and the rows whose attributes $\rho(x)$ are queried by the attacker with identity $\mathrm{GID}_{j}$. This space cannot include the vector $(1,0, \ldots, 0)$, so there is some vector $u^{\prime}$ which is orthogonal to $R$ modulo $p_{2}$ and not orthogonal to $(1,0, \ldots, 0)$. We 
can then write $c w=w^{\prime}+a u^{\prime}$ for some $a$ modulo $p_{2}$ and $w^{\prime}$ in the span of the other basis vectors. We note that $w^{\prime}$ is uniformly distributed in this space, and reveals no information about $a$. The value of the first coordinate of $c w$ modulo $p_{2}$ depends on the value of $a$, but the shares $\delta_{x}$ for $A_{x} \in B$ contain no information about $a$. The only information $\mathcal{A}$ receives about the value of $a$ appears in exponents of the form $\delta_{x}+\gamma_{x} z_{\rho(x)}$, where the $z_{\rho(x)}$ is a new random value each time that appears nowhere else (recall that $\rho$ is constrained to be injective). (We note that these $z_{\rho(x)}$ values modulo $p_{2}$ do not occur in any keys for identities not equal to $\mathrm{GID}_{j}$, since these keys are either normal or semifunctional of type 2 , and hence do not have components in $G_{p_{2}}$.) As long as $\gamma_{x}$ does not equal 0 ( $\gamma_{x}=0$ with only negligible probability), this means that any value of $\delta_{x}$ can be explained by $z_{\rho(x)}$ taking on a particular value. Since $z_{\rho(x)}$ is uniformly random, this means that no information about the value of $a$ modulo $p_{2}$ is revealed. Hence, the value being shared is information-theoretically hidden, and the $\delta_{x}$ 's are properly distributed in the adversary's view.

Though it is hidden from $\mathcal{A}$, the fact that we can only make $\delta_{x}$ shares of 0 is crucial here (i.e. the simulator can only make a nominally semi-functional ciphertext). If $\mathcal{B}$ tried to test the semi-functionality of the $j^{\text {th }}$ key for itself by making a challenge ciphertext the key could decrypt, decryption would succeed regardless of the presence of $G_{p_{2}}$ components, since the $\delta_{x}$ 's are shares of 0 . Hence the simulator would not be able to tell whether the $j^{\text {th }}$ key was semi-functional of Type 1 or normal.

In summary, when $T \in G_{p_{1}}, \mathcal{B}$ properly simulates Game $_{j-1,2}$. When $T \in$ $G_{p_{1} p_{2}}, \mathcal{B}$ properly simulates Game $_{j, 1}$ with probability negligibly close to 1 . Hence, $\mathcal{B}$ can use $\mathcal{A}$ to obtain advantage negligibly close to $\epsilon$ in breaking Assumption 2 .

Lemma 4. Suppose there exists a polynomial time algorithm $\mathcal{A}$ such that Game $_{j, 1} A d v_{\mathcal{A}}-$ Game $_{j, 2} A d v_{\mathcal{A}}=\epsilon$. Then we can construct a polynomial time algorithm $\mathcal{B}$ with advantage $\epsilon$ in breaking Assumption 3.

Lemma 5. Suppose there exists a polynomial time algorithm $\mathcal{A}$ such that Game $_{q, 2} A d v_{\mathcal{A}}-$ Game $_{\text {Final }} A d v_{\mathcal{A}}=\epsilon$. Then we can construct a polynomial time algorithm $\mathcal{B}$ with advantage $\epsilon$ in breaking Assumption 4.

\section{Discussion}

There are multiple ways in which one might extend our work.

Removing the Random Oracle. It would be desirable to remove the need for a random oracle and replace it with a concrete function $H$ mapping identities to group elements. One approach would be to fix a degree $d$ polynomial, $P(x)$, and map identities in $\mathbb{Z}_{N}$ to elements of $G$ by setting $H($ GID $):=g^{P(\text { GID })}$, where $g$ denotes a generator of the group $G$. This approach has previously been employed to obtain large universe constructions for Attributed-Based encryption [30]. The public parameters would then include $\left\{g^{P\left(x_{i}\right)}\right\}$ for $d+1$ points $x_{i}$ so that $H$ (GID) could be computed for any GID by polynomial interpolation. We note that $P(x)$ 
is a $(d+1)$-wise independent function modulo primes, but this will leave the system vulnerable to collusion attacks when $\geq d+1$ users collude. Clearly, this is far from ideal, and we would prefer a better method with stronger security guarantees.

Prime order groups. An interesting direction is create a prime order group variant of our system. Using groups of prime order can potentially lead to more efficient systems (via faster group operations) and security under different assumptions. One approach is to simply use our exact construction except use a group order of one prime (instead of a product of three primes). Applying this setting results in an efficient system that we show to be generically secure in the full version of this paper. However, this construction does not lend itself (to the best of our knowledge) to a proof under a non-interactive assumption.

Another possible approach is to realize the subspaces needed for dual system encryption proofs using vector spaces over prime order groups instead of subgroups. We note that several systems such as BGN encryption [15], GrothOstrovsky-Sahai NIZK proofs [32], traitor tracing [16], and predicate encryption [17,36] were originally developed in the composite order setting, but later variants were developed in prime order groups $31|4833| 3525|26| 37$ | Ideally, a variant would result in security under a simple assumption such as the decision linear assumption.

\section{References}

1. Abdalla, M., Bellare, M., Catalano, D., Kiltz, E., Kohno, T., Lange, T., MaloneLee, J., Neven, G., Paillier, P., Shi, H.: Searchable encryption revisited: Consistency properties, relation to anonymous ibe, and extensions. Journal of Cryptology 21, 350-391 (2008)

2. Abdalla, M., Kiltz, E., Neven, G.: Generalized key delegation for hierarchical identity-based encryption. In: Biskup, J., López, J. (eds.) ESORICS 2007. LNCS, vol. 4734, pp. 139-154. Springer, Heidelberg (2007)

3. Al-Riyami, S., Malone-Lee, J., Smart, N.: Escrow-free encryption supporting cryptographic workflow. Int. J. Inf. Sec. 5, 217-229 (2006)

4. Bagga, W., Molva, R., Crosta, S.: Policy-based encryption schemes from bilinear pairings. In: ASIACCS, pp. 368 (2006)

5. Barbosa, M., Farshim, P.: Secure cryptographic workflow in the standard model. In: Barua, R., Lange, T. (eds.) INDOCRYPT 2006. LNCS, vol. 4329, pp. 379-393. Springer, Heidelberg (2006)

6. Beimel, A.: PhD thesis, Israel Institute of Technology, Technion, Haifa, Israel (1996)

7. Bellare, M., Waters, B., Yilek, S.: Identity-based encryption secure against selective opening attack. In: Ishai, Y. (ed.) TCC 2011. LNCS, vol. 6597, pp. 235-252. Springer, Heidelberg (2011)

8. Bethencourt, J., Sahai, A., Waters, B.: Ciphertext-policy attribute-based encryption. In: IEEE Symposium on Security and Privacy, pp. 321-334 (2007)

${ }^{4}$ Freeman [25] discusses a class of general transformations, although it does not encompass our construction. 
9. Boneh, D., Boyen, X.: Efficient selective-ID secure identity-based encryption without random oracles. In: Cachin, C., Camenisch, J.L. (eds.) EUROCRYPT 2004. LNCS, vol. 3027, pp. 223-238. Springer, Heidelberg (2004)

10. Boneh, D., Boyen, X.: Secure identity based encryption without random oracles. In: Franklin, M. (ed.) CRYPTO 2004. LNCS, vol. 3152, pp. 443-459. Springer, Heidelberg (2004)

11. Boneh, D., Boyen, X., Goh, E.: Hierarchical identity based encryption with constant size ciphertext. In: Cramer, R. (ed.) EUROCRYPT 2005. LNCS, vol. 3494, pp. 440-456. Springer, Heidelberg (2005)

12. Boneh, D., Di Crescenzo, G., Ostrovsky, R., Persiano, G.: Public key encryption with keyword search. In: Cachin, C., Camenisch, J.L. (eds.) EUROCRYPT 2004. LNCS, vol. 3027, pp. 506-522. Springer, Heidelberg (2004)

13. Boneh, D., Franklin, M.: Identity-based encryption from the weil pairing. In: Kilian, J. (ed.) CRYPTO 2001. LNCS, vol. 2139, pp. 213-229. Springer, Heidelberg (2001)

14. Boneh, D., Gentry, C., Hamburg, M.: Space-efficient identity based encryption without pairings. In: Proceedings of FOCS, pp. 647-657 (2007)

15. Boneh, D., Goh, E., Nissim, K.: Evaluating 2-DNF formulas on ciphertexts. In: Kilian, J. (ed.) TCC 2005. LNCS, vol. 3378, pp. 325-341. Springer, Heidelberg (2005)

16. Boneh, D., Sahai, A., Waters, B.: Fully collusion resistant traitor tracing with short ciphertexts and private keys. In: Vaudenay, S. (ed.) EUROCRYPT 2006. LNCS, vol. 4004, pp. 573-592. Springer, Heidelberg (2006)

17. Boneh, D., Waters, B.: Conjunctive, subset, and range queries on encrypted data. In: Vadhan, S.P. (ed.) TCC 2007. LNCS, vol. 4392, pp. 535-554. Springer, Heidelberg (2007)

18. Bradshaw, R., Holt, J., Seamons, K.: Concealing complex policies with hidden credentials. In: ACM Conference on Computer and Communications Security, pp. 146-157 (2004)

19. Camenisch, J., Lysyanskaya, A.: An efficient system for non-transferable anonymous credentials with optional anonymity revocation. In: Pfitzmann, B. (ed.) EUROCRYPT 2001. LNCS, vol. 2045, p. 93. Springer, Heidelberg (2001)

20. Canetti, R., Halevi, S., Katz, J.: A forward-secure public-key encryption scheme. In: Biham, E. (ed.) EUROCRYPT 2003. LNCS, vol. 2656, pp. 255-271. Springer, Heidelberg (2003)

21. Chase, M.: Multi-authority attribute based encryption. In: Vadhan, S.P. (ed.) TCC 2007. LNCS, vol. 4392, pp. 515-534. Springer, Heidelberg (2007)

22. Chase, M., Chow, S.: Improving privacy and security in multi-authority attributebased encryption. In: ACM Conference on Computer and Communications Security, pp. 121-130 (2009)

23. Cheung, L., Newport, C.: Provably secure ciphertext policy abe. In: ACM Conference on Computer and Communications Security, pp. 456-465 (2007)

24. Cocks, C.: An identity based encryption scheme based on quadratic residues. In: Honary, B. (ed.) Cryptography and Coding 2001. LNCS, vol. 2260, pp. 26-28. Springer, Heidelberg (2001)

25. Freeman, D.M.: Converting pairing-based cryptosystems from composite-order groups to prime-order groups. In: Gilbert, H. (ed.) EUROCRYPT 2010. LNCS, vol. 6110, pp. 44-61. Springer, Heidelberg (2010)

26. Garg, S., Kumarasubramanian, A., Sahai, A., Waters, B.: Building efficient fully collusion-resilient traitor tracing and revocation schemes. In: ACM Conference on Computer and Communications Security, pp. 121-130 (2010) 
27. Gentry, C.: Practical identity-based encryption without random oracles. In: Vaudenay, S. (ed.) EUROCRYPT 2006. LNCS, vol. 4004, pp. 445-464. Springer, Heidelberg (2006)

28. Gentry, C., Silverberg, A.: Hierarchical id-based cryptography. In: Zheng, Y. (ed.) ASIACRYPT 2002. LNCS, vol. 2501, pp. 548-566. Springer, Heidelberg (2002)

29. Goyal, V., Jain, A., Pandey, O., Sahai, A.: Bounded ciphertext policy attribute based encryption. In: Aceto, L., Damgård, I., Goldberg, L.A., Halldórsson, M.M., Ingólfsdóttir, A., Walukiewicz, I. (eds.) ICALP 2008, Part II. LNCS, vol. 5126, pp. 579-591. Springer, Heidelberg (2008)

30. Goyal, V., Pandey, O., Sahai, A., Waters, B.: Attribute Based Encryption for FineGrained Access Conrol of Encrypted Data. In: ACM Conference on Computer and Communications Security, pp. 89-98 (2006)

31. Groth, J., Ostrovsky, R., Sahai, A.: Non-interactive zaps and new techniques for nizk. In: Dwork, C. (ed.) CRYPTO 2006. LNCS, vol. 4117, pp. 97-111. Springer, Heidelberg (2006)

32. Groth, J., Ostrovsky, R., Sahai, A.: Perfect non-interactive zero knowledge for np. In: Vaudenay, S. (ed.) EUROCRYPT 2006. LNCS, vol. 4004, pp. 339-358. Springer, Heidelberg (2006)

33. Groth, J., Sahai, A.: Efficient non-interactive proof systems for bilinear groups. In: Smart, N.P. (ed.) EUROCRYPT 2008. LNCS, vol. 4965, pp. 415-432. Springer, Heidelberg (2008)

34. Horwitz, J., Lynn, B.: Toward hierarchical identity-based encryption. In: Knudsen, L.R. (ed.) EUROCRYPT 2002. LNCS, vol. 2332, pp. 466-481. Springer, Heidelberg (2002)

35. Iovino, V., Persiano, G.: Hidden-vector encryption with groups of prime order. In: Galbraith, S.D., Paterson, K.G. (eds.) Pairing 2008. LNCS, vol. 5209, pp. 75-88. Springer, Heidelberg (2008)

36. Katz, J., Sahai, A., Waters, B.: Predicate encryption supporting disjunctions, polynomial equations, and inner products. In: Smart, N.P. (ed.) EUROCRYPT 2008. LNCS, vol. 4965, pp. 146-162. Springer, Heidelberg (2008)

37. Lewko, A., Okamoto, T., Sahai, A., Takashima, K., Waters, B.: Fully secure functional encryption: Attribute-based encryption and (hierarchical) inner product encryption. In: Gilbert, H. (ed.) EUROCRYPT 2010. LNCS, vol. 6110, pp. 62-91. Springer, Heidelberg (2010)

38. Lewko, A., Waters, B.: Decentralizing attribute-based encryption. Cryptology ePrint Archive, Report 2010/351 (2010), http://eprint.iacr.org/

39. Lewko, A., Waters, B.: New techniques for dual system encryption and fully secure HIBE with short ciphertexts. In: Micciancio, D. (ed.) TCC 2010. LNCS, vol. 5978, pp. 455-479. Springer, Heidelberg (2010)

40. Lin, H., Cao, Z., Liang, X., Shao, J.: Secure threshold multi authority attribute based encryption without a central authority. In: Chowdhury, D.R., Rijmen, V., Das, A. (eds.) INDOCRYPT 2008. LNCS, vol. 5365, pp. 426-436. Springer, Heidelberg (2008)

41. Miklau, G., Suciu, D.: Controlling access to published data using cryptography. In: VLDB 2003, pp. 898-909 (2003)

42. Müller, S., Katzenbeisser, S., Eckert, C.: Distributed attribute-based encryption. In: Lee, P.J., Cheon, J.H. (eds.) ICISC 2008. LNCS, vol. 5461, pp. 20-36. Springer, Heidelberg (2009)

43. Müller, S., Katzenbeisser, S., Eckert, C.: On multi-authority ciphertext-policy attribute-based encryption. Bulletin of the Korean Mathematical Society 46(4), 803-819 (2009) 
44. Ostrovksy, R., Sahai, A., Waters, B.: Attribute Based Encryption with NonMonotonic Access Structures. In: ACM Conference on Computer and Communications Security, pp. 195-203 (2007)

45. Sahai, A., Waters, B.: Fuzzy Identity-Based Encryption. In: Cramer, R. (ed.) EUROCRYPT 2005. LNCS, vol. 3494, pp. 457-473. Springer, Heidelberg (2005)

46. Shamir, A.: Identity-based cryptosystems and signature schemes. In: Blakely, G.R., Chaum, D. (eds.) CRYPTO 1984. LNCS, vol. 196, pp. 47-53. Springer, Heidelberg (1985)

47. Shi, E., Bethencourt, J., Chan, H., Song, D., Perrig, A.: Multi-dimensional range query over encrypted data. In: IEEE Symposium on Security and Privacy (2007)

48. Shi, E., Bethencourt, J., Chan, H.T.-H., Xiaodong Song, D., Perrig, A.: Multidimensional range query over encrypted data. In: IEEE Symposium on Security and Privacy, pp. 350-364 (2007)

49. Shi, E., Waters, B.: Delegating capabilities in predicate encryption systems. In: Aceto, L., Damgård, I., Goldberg, L.A., Halldórsson, M.M., Ingólfsdóttir, A., Walukiewicz, I. (eds.) ICALP 2008, Part II. LNCS, vol. 5126, pp. 560-578. Springer, Heidelberg (2008)

50. Shoup, V.: Lower bounds for discrete logarithms and related problems. In: Fumy, W. (ed.) EUROCRYPT 1997. LNCS, vol. 1233, pp. 256-266. Springer, Heidelberg (1997)

51. Smart, N.: Access control using pairing based cryptography. In: Joye, M. (ed.) CT-RSA 2003. LNCS, vol. 2612, pp. 111-121. Springer, Heidelberg (2003)

52. Waters, B.: Efficient identity-based encryption without random oracles. In: Cramer, R. (ed.) EUROCRYPT 2005. LNCS, vol. 3494, pp. 114-127. Springer, Heidelberg (2005)

53. Waters, B.: Dual system encryption: realizing fully secure ibe and hibe under simple assumptions. In: Halevi, S. (ed.) CRYPTO 2009. LNCS, vol. 5677, pp. 619-636. Springer, Heidelberg (2009)

54. Waters, B.: Ciphertext-policy attribute-based encryption: An expressive, efficient, and provably secure realization. In: Catalano, D., Fazio, N., Gennaro, R., Nicolosi, A. (eds.) PKC 2011. LNCS, vol. 6571, pp. 53-70. Springer, Heidelberg (2011) 